\title{
Characterization of thermotropic liquid crystalline polymers
}

\author{
Robert W. Lenz \\ Chemical Engineering Department, University of Massachusetts, \\ Amherst, Mass. 01003 U.S.A.
}

\begin{abstract}
The characterization of thermotropic, main chain liquid crystalline polymers for the type, thermal and thermodynamic properties, order, and degree of liquid crystallinity of the anisotropic phase is described through the use of the following methods: (1) polarized light microscopy, (2) wide angle X-ray diffraction, (3) differential scanning calorimetry, (4) small angle light scattering, (5) electron microscopy and electron diffraction, (6) magnetic resonance procedures for order parameter measurements, and (7) melt rheology. Polymers which form either or both nematic and smectic phases are described.
\end{abstract}

\section{INTRODUCTION}

The systematic study of thermotropic, main chain, liquid crystalline polymers began only about ten years ago, and the first publications in the open literature on such polymers were apparently those by Roviello and Sirigu in 1975 (ref. 1) and by Jackson and Kuhfuss in 1976 (ref. 2), both on polyesters. Hence, it is not surprising that our ability to characterize the physical behavior and properties of these polymers and our understanding of their structure-property relationships is still quite superficial. Indeed, we might draw the analogy that our understanding of the liquid crystalline state in polymers today is at a level about equivalent to our understanding of the crystalline state of polymers in the 1940 s, which was the period before the structures of spherulites or the possibility of folded chain crystallization were known. However, today we have many different types of characterization methods that were not available at that time, and these methods are now being rapidly applied to elucidating the various structural levels and types of liquid crystallinity in polymer melts and solutions. This report will be concerned only with the former, the melt or thermotropic liquid crystalline state, but the thermotropic and lyotropic states have much in common for main chain, liquid crystalline, LC, polymers.

\section{CHARACTERIZATION OF LC POLYMERS}

When a new polymer is prepared in our laboratories, and by other investigators, a standard set of characterization methods is immediately applied to determine if the polymer forms a thermotropic melt, what type of liquid crystalline phase is formed (nematic or smectic) and at what temperatures the phase changes occur (including melting, smectic to nematic, isotropization or clearing, deisotropization, and recrystallization). For specific polymers of particular interest, additional methods are applied including: (1) small angle light scattering and transmission electron microscopy to investigate the morphology of the LC state; (2) wide angle X-ray diffraction to more fully characterize the nematic and smectic states; and (3) melt viscosity and rheological behavior for dynamic morphological investigations on homopolymers and for observing effects of variations in the "degree of crystallinity" in copolymers containing both mesogenic and nonmesogenic units, and other more specialized methods.

The first indication of the ability of a polymer to melt or soften directly into a liquid crystalline state, however, can often be made by a simple visual observation of a sample on a hot plate. Most such polymers will take on a pearly or opalescent appearance when their LC melts are sheared or stirred. Furthermore, we have observed that for copolymers containing varying amounts of mesogenic and nonmesogenic units which form two-phase melts (nematic and isotropic), the brightness of their "stir opalescence" can be taken as a semiquantitative indication of their "degree of liquid crystallinity" (ref. 3).

\section{Microscopy}

The characterization of liquid crystals by polarized light microscopy is the simplest method available and, whenever possible, it is carried out in the initial stages of an investigation on new polymers because thermal analyses alone do not indicate the type of phase 
formed and can often be misleading. The appearance of a particular "texture" of the melt is usually dependent on the structure of the mesophase, and, therefore, it is often possible to directly identify the type of mesophase present by this method. Several books are available with photomicrographs of the textures of various types of liquid crystal phases for low molecular compounds, and these can serve as guidelines for identifying polymer phases (ref. 4).

Texture observation of low molecular weight compounds is usually much easier, however, than that of their polymeric counterparts because their lower melt viscosities enable the quicker appearance of identifiable melt structures. Polymeric materials can take minutes or hours to show recognizable textures, and in that time period the polymers can degrade or crosslink. Indeed, some polymers either show no definite texture, possibly because of their high molecular weight, and consequently they may be simply identified as nematic (refs. 5, 6), or the sample may not develop the equilibrium texture and be incorrectly identified, as happened in our laboratory recently (ref. 7). Hence, this type of identification must be considered to be only provisional until verified by X-ray methods.

In general, however, the textures exhibited by polymers are identical to those of low molecular weight compounds. The threaded Schlieren texture, typical of nematics, and the focal-conic or fan-shaped textures typical of smectics, are the ones most often cited in the literature (ref. 8). Cholesteric polymers, like their low molecular weight counterparts, also produce observations of "oily streaks" and parallel disclinations (ref. 9).

A more quantified approach to microscopy involves measuring the depolarized light intensity with a photocell and plotting this quantity as a function of temperature. This approach is particularly useful in determining the clearing transition when rather broad transitions, quite usual for polymers, are obtained (ref. 10).

\section{X-Ray Diffraction}

Studies of thermotropic polymers by wide-angle X-ray diffraction, WAXD, in the liquid state can be very useful in the identification of the structure of the liquid crystalline melt because microscopy experiments cannot always be counted on to produce an unambiguous determination of the type of mesophase present. In WAXD patterns, nematic structures form a diffuse ring at $4-5 \AA$ on a flat film diffractogram, which arises from the interchain spacings (ref. 11). Cholesteric mesophases in general resemble the nematic mesophase when observed by both small-angle X-ray diffraction, SAXD, and WAXD. The smectic mesophase, in contrast, produces both diffuse rings at $4-5 \AA$ and sharp rings at a distance generally, but not necessarily, equal to the repeat length of the monomer unit, between 15 and $50 \AA$ (refs. $8,12,13)$.

Recently we studied several polymers in a series containing an aromatic ester triad unit and polymethylene flexible spacers of varying lengths (see Series I below) that were identified by texture observation as being smectic (ref. 8). X-ray diffraction patterns showed a spacing at approximately $5 \AA$ for all three and spacings at 28,31 , and $33 \AA$ for the polymers with 9, 10, and 12 (ref. 14) methylene groups in the spacer, respectively. The texture of the polymer with the nonamethylene spacer was similar to that of a smectic C phase in which the mesogen is tilted with respect to the layers, and such a structure would be consistent with the observed interlayer distance of $28 \AA$, which is less than that expected for the repeating unit in the fully extended conformation. On the other hand, the polymer 12 methylene groups had a texture indicative of a smectic A phase, but the $D$ spacing of $33 \AA$ is not sufficient to allow for a perpendicular alignment with a full trans conformation of its repeating unit (ref. 8 ). The basis of this discrepancy is still unknown.

\section{Thermal Analysis}

In addition to characterization of the mesophase by microscopy and X-ray diffraction, the most common technique for studying liquid crystalline compounds and polymers is by thermal analysis. This technique both reveals the transition temperatures and gives a measure of the heats and entropies of transition. A large amount of literature exists concerning the thermal behavior of low molecular weight compounds, and most studies of LC polymers and model LC compounds have been performed using a DSC or DTA instrument.

The melting temperatures of polymeric materials can be greatly affected by the sample history, but it is now commonly agreed that the isotropization or clearing transition is much less subject to effects of thermal treatment and show little undercooling. If it can be assumed, therefore, that the clearing transition is close to an equilibrium process, then the entropy of this transition, $\Delta S_{i}$, can be calculated from the clearing temperature and from the enthalpy of the transition. Values of $\Delta S_{i}$ should give some indication of the order present in the system if the isotropic state is assumed to have equal disorder in all systems (ref. 15), and the order should be related to the type of LC phase. 
Values of $\Delta \mathrm{S}_{j}$ and $\Delta \mathrm{H}_{j}$ have been determined for many different main chain LC polymers, particularly those with polymethylene spacers, and correlated with the type of mesophase formed (refs. 16, 17). A remarkable feature of most of these polymers is that, when the homologous series was studied, an even-odd effect in the entropy of clearing was observed which was quite similar to the even-odd properties of both the melting and clearing transition temperatures. However, the interpretation of the variation in relative values of $\Delta S_{i}$ along such a series can be complicated by changes in the type of mesophase formed from nematic to smectic in passing from an even to an odd number of methylene units, as discussed below. It should also be noted that the values of $\Delta S_{i}$ for model compounds are generally much lower than those for polymers, which suggests that the order present in a polymeric mesophase may be much higher than is found in a model system and that a critical interplay exists between the mesogenic unit and the flexible spacer.

Two interesting series of polymers studied in our laboratory for the effect of the flexible spacer structure and length on the mesophase type and order were the following $(r e f s .7,10)$ :

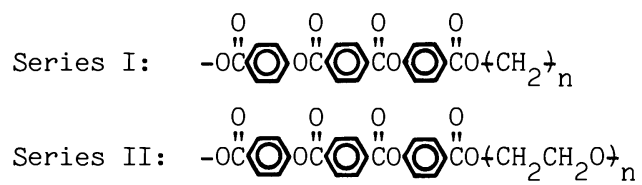

Both types of polymers contain the same mesogen, and within each series polymers with flexible spacers of different lengths were prepared and characterized. For Series I with the polymethylene spacers, individual polymers containing repeating units with $\mathrm{n}=2-10$ and 12 were prepared with the results given in Table 1 (ref. 7). For Series II, a more limited series was evaluated as shown in Table 2 (ref. 10). Polymer samples (5) to (8) in this table, with $\mathrm{n}$ values greater than 4, were prepared from poly (ethylene glycol) oligomers containing a distribution of units with an average value of $\mathrm{n}$ as indicated, while those with $\mathrm{n}=1-4$, for Polymers (1)-(4) in Table 2, were prepared from pure glycols containing the exact number of ethyleneoxy units indicated.

TABLE 1. Properties of Series I polymers with polymethylene spacers

\begin{tabular}{|c|c|c|c|c|}
\hline \multirow[t]{2}{*}{$n$} & $\mathrm{~T}_{\mathrm{m}}$ & $\mathrm{T}_{\mathrm{i}}$ & $\Delta \mathrm{T}$ & $\Delta S_{i}$ \\
\hline & \multicolumn{3}{|c|}{$\left({ }^{\circ} \mathrm{C}\right)$} & $\mathrm{cal} / \mathrm{mol}-{ }^{\circ} \mathrm{K}$ \\
\hline 2 & 342 & $365^{a}$ & 23 & -- \\
\hline 3 & 240 & $315^{a}$ & 75 & -- \\
\hline $4 \mathrm{~h}$ & 285 & $345^{\mathrm{a}}$ & 60 & -- \\
\hline $5^{0}$ & (190) & 267 & (77) & 2.7 \\
\hline 6 & 227 & 275 & 48 & 1.2 \\
\hline 7 & 176 & 253 & 77 & 3.1 \\
\hline 8 & 197 & 220 & 23 & 2.3 \\
\hline 9 & 174 & 233 & 63 & 2.7 \\
\hline 10 & 220 & 267 & 47 & 3.6 \\
\hline 12 & 212 & 245 & 33 & 3.6 \\
\hline
\end{tabular}

${ }^{a}$ Decomposition temperature.

$\mathrm{b}_{\text {Transition observed upon first heating }}$ only.
TABLE 2. Properties of Series II polymers with poly(ethylene glycol) spacers

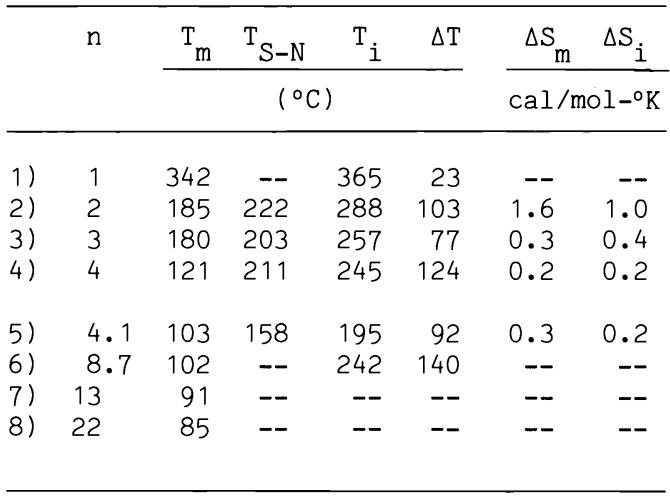

The polymers of Series I were originally reported by us to form nematic phases on melting for $\mathrm{n}=2-8$ and smectic phases for $\mathrm{n}=9,10$ and 12 (ref. 7). That report was based on incorrect interpretations of the textures of their liquid crystalline phases as seen on the polarizing microscope. A more careful reexamination has revealed that polymers with $\mathrm{n}=3,5,7$ and 8 all form smectic phases on melting, while only those with $\mathrm{n}=4$ and 6 melt into a nematic phase, and that with $\mathrm{n}=2$ is difficult to categorize with any certainty (refs. 16, 17). These visual observations have also been verified by wide angle X-ray diffraction. 
A typical DSC thermogram, that for the polymer with $\mathrm{n}=10$, is shown in Figure 1 (ref. 17). For the sample of Figure 1, the narrower melting, $\mathrm{T}_{\mathrm{m}}$, and broader isotropization, $\mathrm{T}_{\mathrm{i}}$, endothermic peaks are seen in the heating cycle (upper plot), while the smaller deisotropization, $\mathrm{T}_{d}$, and sharper recrystallization, $\mathrm{T}_{C}$, exothermic peaks are seen in the cooling cycle (lower plot).

The value of $\Delta S_{i}$ for the polymer in Fig. 1, which was calculated from the area of the broad $\mathrm{T}_{i}$ endotherm shown, is given in Table 1 along with those of the other polymers in Series I. The higher values of $\Delta S_{i}$ for the polymers with $n=5,7,9,10$ and 12 support the suggestion, based on the observation of their textures on the polarizing microscope, that these form more highly organized smectic phases, as compared to that with $\mathrm{n}=6$, which appears to form the nematic state.

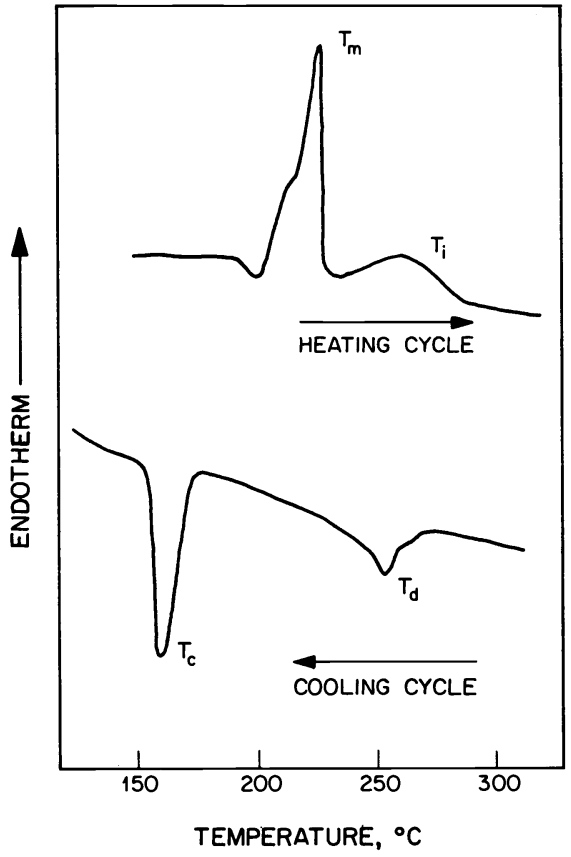

Fig. 1. DSC thermogram of Series I polymer with a decamethylene spacer $(n=10)$, which forms a smectic phase on melting.

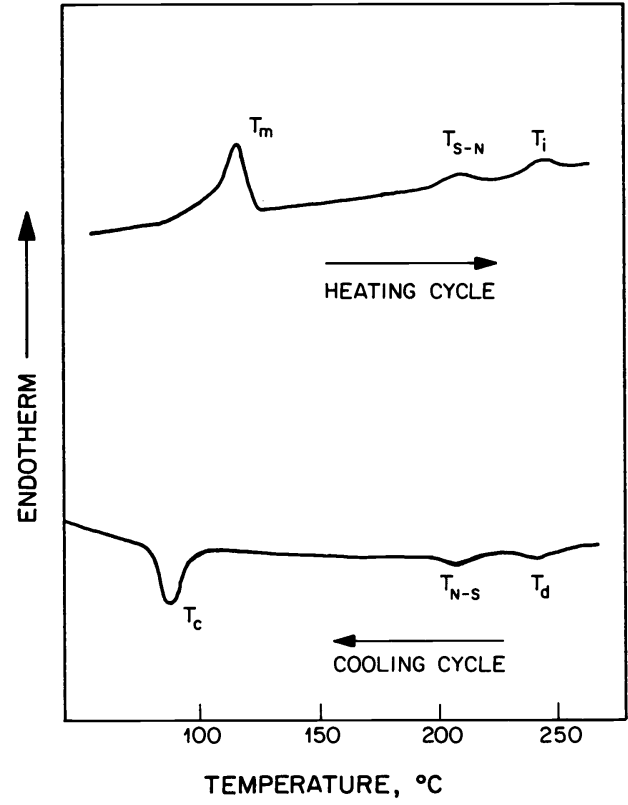

Fig. 2. DSC thermogram of Series II polymer with a tetraethyleneoxy spacer $(n=4)$, which forms both smectic and nematic phases sequentially.

The polymers of Series II are of particular interest because the lower members of this series, Polymers (1)-(5) of Table 2 with $\mathrm{n}=2-4$, form both smectic and nematic phases, and two endothermic peaks were present in their DSC thermograms above $\mathrm{T}_{\mathrm{m}}$, as shown in Fig. 2 for Polymer (4). For these polymers, $\mathrm{T}_{\mathrm{m}}$ represents the melting transition into a smectic phase, and $\mathrm{T}_{\mathrm{S}-\mathrm{N}}$ represents the smectic to nematic transition temperature in Table 2 . The lone exception in Table 2 is Polymer (6), with $\mathrm{n}=8.7$, which melts directly into a nematic phase. Polymers (7) and (8) do not form a liquid crystalline phase on melting. Polymers (2) to (4) were also observed with the polarizing microscope to spontaneously undergo a homeotropic orientation within their smectic phases.

Thermal analysis by either DSC or DTA is especially useful for observing monotropic behavior in LC polymers; that is, for polymers in which $\mathrm{T}_{i}$ is below $\mathrm{T}_{\mathrm{m}}$. In these cases, the formation of the liquid crystalline phase is possible only on cooling from the isotropic melt, unless crystallization can be prevented. An example of such a case is seen for the DSC thermogram in Fig. 3 of the following polymer with a dyad ester mesogen and a hexamethylene spacer (ref. 18):

$$
\text { - }
$$

This polymer melts at $220^{\circ} \mathrm{C}$ directly into an isotropic phase, and only a melting endotherm is present in the thermogram in Fig. 3. However, on cooling, a nematic phase forms first, beginning at about $180^{\circ} \mathrm{C}$, before the delayed recrystallization occurs beginning at $160^{\circ} \mathrm{C}$. The smaller deisotropization exothermic peak is clearly seen in the cooling cycle in Fig. 3 at the higher temperature than that of the recrystallization exothermic peak because of the undercooling for the latter. 


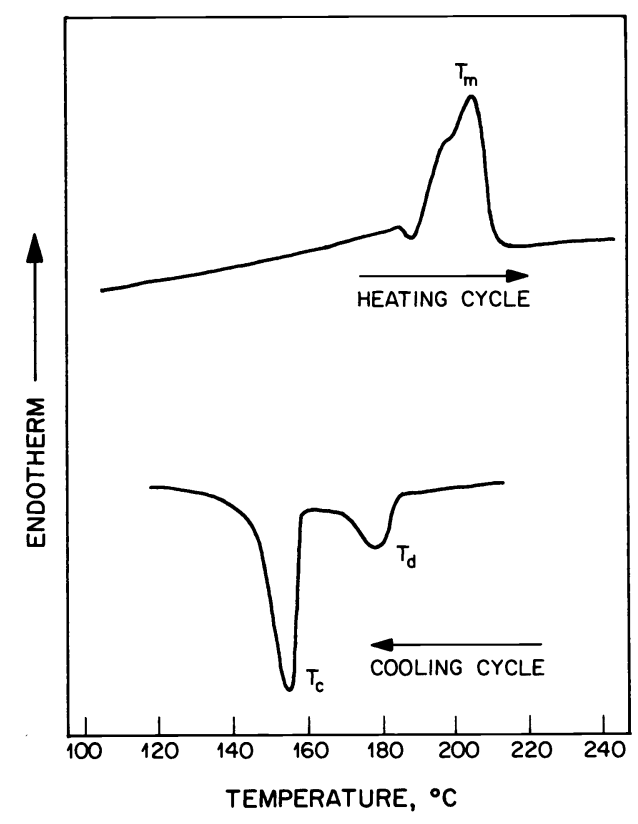

Fig. 3. DSC thermogram of a polymer with a dyad ester mesogen and a hexamethylene spacer showing monotropic behavior.

Small Angle Light Scattering

Small angle light scattering, SALS, methods are applied to polymers to characterize their tertiary order or morphology at dimensions in the region of 500 to $10,000 \AA$. This technique has proven to be particularly useful, for example, in the study of the spherulitic crystalline morphology (ref. 19).

In the nematic state, and probably in the smectic state too, LC compounds and polymers apparently organize into domains of approximately one micron in size, but very little is known about either the shape of these domains, or the interrelationships between neighboring domains, or the effect of environment (particularly temperature and shear) on their structures. Therefore, SALS is being applied by Stein and coworkers, who helped to pioneer this field, to investigate the effects of temperature on the domain morphology of thermotropic LC polymers by using $\mathrm{H}_{\mathrm{v}}$ and $\mathrm{V}_{\mathrm{v}}$ polarization with photographic and photometric methods of analysis (ref. 20).

For nematic polyesters, at least, it has been observed that approximately circular scattering patterns were obtained and that the scattering intensity was azimuthally independent, with $\mathrm{H}_{\mathrm{v}}$ intensities being comparable to $\mathrm{V}_{\mathrm{v}}$, over most or all of the temperature range of the nematic mesophase. These observations indicate that the macroscopic scattering elements, the domains, were approximately spherical in shape and that there was little or no correlation between director axes in neighboring domains. Similar preliminary results have recently been obtained for a smectic mesophase of an LC polymer.

For one of the nematic polymers studied by Stein and coworkers, as the temperature approached the clearing point, the pattern changed spontaneously from a circular shape to one characteristic of the scattering from anisotropic, rod-like elements with correlated orientation fluctuations. This transition was reproducible and reversible, but no satisfactory explanation of this behavior can be offered at present (ref. 20).

Electron Microscopy and Electron Diffraction

Electron microscopy and diffraction have been applied by Donald and coworkers to reveal and investigate an unusual banded structure formed by LC polymers on quenching after being oriented in the nematic state (ref. 21). Thomas and coworkers (ref. 22) have also recently applied this method to morphological investigations of oriented thin films of two LC polyesters of the following structure prepared in our laboratory:

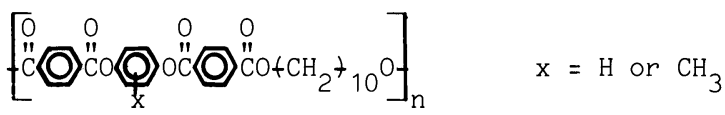


Layer lines with specific spacings were observed in the solid polymer by electron microscopy which indicated that the polymers formed an almost fully extended conformation during orientation, and multiple equatorial reflections observed by electron diffraction suggested that biaxial chain packing was present in the oriented nematic state of these polymers before quenching. Equatorial dark field and polarized light microscopy also revealed the presence of an alternating, transverse band structure in these films. For the oriented polymers, therefore, it was concluded that the LC state did not contain a domain structure but consisted instead of a continuous phase containing various types of disclinations (ref. 22).

A similar morphology was observed by Shiru and coworkers (ref. 23) in oriented films of a closely related polyester, of the following structure, which was also prepared in our laboratory (refs. 24, 25):

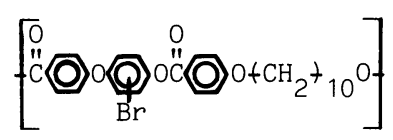

Films of this polymer were characterized by a combination of polarized light microscopy, SALS, and polarized infrared spectroscopy to determine the nature of the alignment of the polymer chains, which was responsible for the banded or striped morphology observed. It was concluded from these observations that the stripes were composed of fibrils arranged in a zig-zag manner containing polymer chains packed in a highly ordered, parallel alignment at an angle of $\pm 26^{\circ}$ to the orientation direction (ref. 23).

\section{Order Parameter Measurements}

The molecular order in LC polymers is generally described in terms of two order parameters, which characterize the orientation of the repeating units within a molecular domain (microorder) and the macroscopic alignment of the domains (macro-order). The measurement of these parameters is important for relating physical properties of liquid crystal polymers to their molecular structure.

Various methods have been used to measure order parameters, including the use of refractive index and dielectric and magnetic susceptibilities, but these methods require knowledge of the corresponding molecular quantities of the polymer on which the interpretations are based (ref. 26). It is of considerable interest, therefore, to develop other experimental methods which do not depend on such information, and in cooperation with Kothe, we have studied the use of electron spin probes in polymers to measure liquid crystalline order from the temperature and angular dependence of their electron spin resonance (ESR) spectra. For this purpose a cholestane probe containing a nitroxide free radical was dissolved in the same main chain liquid crystal polyester as that used by Shiru and coworkers in their studies, which are described above. This probe is stable up to $180^{\circ} \mathrm{C}$ in the polymer melt and, because of its elongated, rodlike shape, it is believed to take on the orientation of the mesogenic units in the polymer backbone. If so, then the ESR line shapes should give valuable information about the micro- and macro-order of the polymer.

Analysis of the line shapes obtained in our study gave the detailed information expected about the order and dynamics of the nematic state of the polyester (ref. 27). The microorder parameter was found to be 0.81 , which is considerably larger than the values generally observed in low molecular weight nematogens.

Subsequently, we also carried out a deuteron NMR analysis of specially prepared deuterated samples of a closely related polyester (ref. 28), and this method verified that the data from the cholestane probe reliably reflected the local chain order. The macro-order parameter determined by this method was essentially 0 , which supports the proposal of a domain morphology for the nematic state in which the polymer chains are highly ordered on a molecular level but in which the director axes of the domains are randomly distributed.

A uniform alignment of the domains could be obtained, however, by extruding this polymer, and this alignment was clearly indicated by their ESR spectra, which were taken below the melting point. A drastic spectral change was observed, and the line shapes clearly showed that extrusion of the polymer in its liquid crystal state produced highly oriented fibers. A detailed analysis, based on spectral simulations, yielded a micro-order parameter of 0.81 , as before, but now practically all of the domains were aligned with a macro-order parameter of 0.9 . Thus, the micro- and macro-orders of this thermotropic main chain polymer could be determined independently. The observed micro-order parameter was also in agreement with order parameters obtained from X-ray diffraction and proton NMR studies (refs. 29-31).

Deuteron NMR studies using multiple pulse dynamic NMR spectroscopy were also carried out on the following types of deuterated polymers, in which the Roman numerals, I-III, refer to different polymer samples of the same molecular structure deuterated at different 
positions (ref. 32):

$$
[\text { (O) }
$$

In this study, computer simulations of the spectra, based on a comprehensive model, provided information on the orientation distributions and conformations of the polymer chains and also on the correlation times of the various motions in the nematic state (ref. 33). The micro-order parameter was found to be 0.85 , again in very good agreement with the ESR and NMR studies described above. In addition, it was found that the polymethylene flexible spacer took on a highly extended conformation as evidenced by a trans population of 0.8 . This degree of micro-order was retained when the polymer was cooled to temperatures below its melting and glass transitions.

\section{Rheological Properties}

The rheology of low molecular weight thermotropic compounds has been a subject of considerable theoretical and experimental analysis (refs. 34, 25). In general, liquid crystals are easily oriented by surfaces, by electromagnetic fields and by mechanical stress or shear, and the degree of orientation, in turn, affects their melt viscosity. The rheological behavior of a liquid crystal is known to be greatly dependent on the nature and also on the texture of its mesophase for both low molecular weight compounds and polymers. Furthermore, the relative values of the melt viscosities as a function of composition for copolymers which contain both mesogenic and nonmesogenic units can be used as a measure of the "degree of liquid crystallinity" in such systems in which both anisotropic LC and isotropic phases are present (ref. 35).

Other characteristic properties of nematic polymers in the melt are the lack of die swell (elastic recovery), very long relaxation times, the critical role of shear history and thermal history on rheological behavior, and unusual yield stress behavior, all of which have been attributed to the presence of domains in nematic melts which have different director orientations (ref. 36). However, again the quantitative study of the rheology of liquid crystal polymers is in its infancy and much more research is needed before one can draw any conclusions based on specific models or theories. Of course, an understanding of the rheological properties and physical states of the liquid crystal phase of polymers is extremely important, not only for theoretical interest but also for the optimization of processing conditions and for the development of maximum physical and mechanical properties in the application of such polymers.

Acknowledgement

The investigations on liquid crystalline polymers in the author's laboratory have been supported by the National Science Foundation, the Office of Naval Research, and the Petroleum Research Fund.

\section{REFERENCES}

1. A. Roviello and A. Sirigu, J. Polym. Sci., Polym. Letters, 13, 455 (1975).

2. W. J. Jackson and H. F. Kuhfuss, J. Polym. Sci., Polym. Chem. Ed., 14, 2043 (1976).

3. R. W. Lenz and J.-I. Jin, Macromolecules, 14, 1405-1411 (1981).

4. D. Demus and L. Richter, Textures of Liquid Crystals, Verlag Chemie, New York (1978).

5. L. Strzelecki and D. van Luyen, Europ. Polym. J., 16, 299 (1980).

6. A. Blumstein, K. Sivaramakrishnan, S. B. Clough and R. B. Blumstein, Mol. Cryst. Liq. Cryst., 49 (Letters), 255 (1979).

7. C. Ober, J.-I. Jin and R. W. Lenz, Polym. J. (Japan), 14, 9 (1982).

8. C. Ober, J.-I. Jin and R. W. Lenz, Makromol. Chem., Rapid Commun., 4 , 49 ( 1983).

9. H.-J. Park, J.-I. Jin and R. W. Lenz, Polymer, in print.

10. G. Galli, E. Chiellini, C. Ober and R. W. Lenz, Makromol. Chem., 183, 2693 (1982).

11. A. DeVries, Mol. Cryst. Liq. Cryst., 10, 219 (1970).

12. L. Liebert, L. Strzelecki, D. van Luyen and A. M. Levelut, Europ. Polym. J., 1771 (1981).

13. A. Blumstein, K. Sivaramakrishnan, R. B. Blumstein and S. B. Clough, Polymer, 23, 47 (1982).

14. P. Hahn, University of Massachusetts, private communication (1984).

15. J. F. Johnson and R. S. Porter, eds., Liquid Crystals and Ordered Fluids, Plenum Press, New York (1974).

16. B. Millaud, A. Thiery and A. Skoulios, Mol. Cryst. Liq. Cryst., 41 (Letters), 263 (1978).

17. C. K. Ober, J.-I. Jin, Q. Zhou and R. W. Lenz, Adv. in Polym. Sci., 59, 103 (1984). 
18. C. Ober, R. W. Lenz, G. Galli and E. Chiellini, Macromolecules, 16, 1034-1036 (1983).

19. R. S. Stein and J. S. Higgins, J. Appl. Cryst., $\overline{11,346(1978)}$.

20. S. A. Jabarin and R. S. Stein, J. Phys. Chem., 77, 399 (1973); 77, 409 (1973).

21. A. M. Donald and A. H. Windle, Polymer, 25, 1235 (1984).

22. E. L. Thomas and B. A. Wood, University of Massachusetts, presentation at Faraday Society Meeting, Cambridge, England (April 1985).

23. H. Shiru, X. Mao, J. Li, B. Qian and R. W. Lenz, J. Polym. Sci., Polym. Phys. Ed., in print.

24. Q.-F. Zhou and R. W. Lenz, J. Polym. Sci., Polym. Chem. Ed., 21, 3313-3320 (1983).

25. S. Antoun, R. W. Lenz and J.-I. Jin, J. Polym. Sci., Polym. Chem. Ed., 19, 1901 (1981).

26. E. T. Samulski and D. B. DuPre, Adv. Liq. Crystallogr., 4, 121-145 (1979).

27. K. Mueller, K.-H. Wassmer, R. W. Lenz and G. Kothe, J. Polym. Sci., Polym. Lett. Ed., 21, 785-789 (1983).

28. K. Mueller, B. Hisgen, H. Ringsdorf, R. W. Lenz and G. Kothe, "Nuclear Spinlabel Studies of Liquid Crystal Polymers," in Recent Advances in Liquid Crystalline Polymers, L. Chapoy, Ed., Applied Science Publishers (1984).

29. G. Kothe and T. E. Berthold, Mol. Phys., 40, 1441-1451 (1980).

30. L. Liebert, L. Strzelecki, D. Van Luyen and A. M. Levelut, Eur. Polym. J., 17, 71-75 (1981).

31. A. Blumstein, S. Vilasagar, S. Ponrathnam, S. B. Clough and R. B. Blumstein, J. Polym. Sci., Polym. Phys. Ed., 20, 877-892 (1982).

32. K. Mueller, C. Eisenbach, B. Hisgen, H. Ringsdorf, A. Schneller, R. W. Lenz and G. Kothe, to be published.

33. K. Mueller, B. Hisgen, H. Ringsdorf, R. W. Lenz and G. Kothe, Mol. Cryst. Liq. Cryst., submitted for publication.

34. K.-H. Wassmer, Ph.D. Thesis, University of Freiburg (1982).

35. V. Nicely, Eastman Kodak Co., to be published.

36. K. Mueller, E. Ohmes, C. Eisenbach, B. Hisgen, H. Ringsdorf, A. Schneller, R. W. Lenz and G. Kothe, to be published. 